\title{
Controlling Spin Qubits in Quantum Dots
}

\author{
Hans-Andreas Engel \\ Department of Physics and Astronomy, University of Basel, Klingelbergstrasse 82, \\ CH-4056 Basel, Switzerland \\ L.P. Kouwenhoven \\ Department of NanoScience and ERATO Mesoscopic Correlation Project, Delft \\ University of Technology, P.O. Box 5046, 2600 GA Delft, The Netherlands \\ Daniel Loss \\ Department of Physics and Astronomy, University of Basel, Klingelbergstrasse 82, \\ CH-4056 Basel, Switzerland \\ C.M. Marcus \\ Department of Physics, Harvard University, Cambridge, Massachusetts 02138, \\ USA
}

\begin{abstract}
We review progress on the spintronics proposal for quantum computing where the quantum bits (qubits) are implemented with electron spins. We calculate the exchange interaction of coupled quantum dots and present experiments, where the exchange coupling is measured via transport. Then, experiments on single spins on dots are described, where long spin relaxation times, on the order of a millisecond, are observed. We consider spin-orbit interaction as sources of spin decoherence and find theoretically that also long decoherence times are expected. Further, we describe the concept of spin filtering using quantum dots and show data of successful experiments. We also show an implementation of a read out scheme for spin qubits and define how qubits can be measured with high precision. Then, we propose new experiments, where the spin decoherence time and the Rabi oscillations of single electrons can be measured via charge transport through quantum dots. Finally, all these achievements have promising applications both in conventional and quantum information processing.
\end{abstract}

Keywords: spin qubits, coupled quantum dots, spin filter, spin read out

\section{Introduction}

The spin degree of freedom promises many applications in electronics [1, 2, 3. Prominent experiments have shown injection of spin-polarized currents into semiconductor material [4, 5], long spin dephasing times in semiconductors (approaching microseconds) [6], ultrafast coherent spin manipulation [7, as well as phase-coherent spin transport over distances of up to $100 \mu \mathrm{m}$ [6]. Irrespective of spin, the charge of the electrons can be used to control single electrons by confining them in quantum dot structures, which leads to striking effects in the Coulomb blockade regime [8]. The Loss and DiVincenzo proposal [9] combines

(C) 2018 Kluwer Academic Publishers. Printed in the Netherlands. 
these two fields of research and uses the spin of electrons confined on quantum dots as spin qubits for quantum computation. This proposal comprises two-qubit quantum gates relying on the exchange interaction of coupled quantum dots and comprises spin-to-charge conversion for efficient read-out schemes, satisfying all theoretical requirements for quantum computing. This quantum computer proposal, based on exchange interaction, can be mapped from electron spins on dots to nuclear spins of $\mathrm{P}$ atoms in $\mathrm{Si}$, as shown by Kane [10] (see article in this issue).

The spin qubit proposal [9] addresses the central issues for building a quantum computer. However, for a concrete implementation of spin qubits, a more detailed theoretical and experimental understanding of spins on quantum dots is required. This demand has led to many new theoretical and experimental investigations on quantum dots, which also address interesting aspects of physics on their own. In this article we will review some of these recent results.

\subsection{Quantum Dots}

In this article we consider semiconductor quantum dots. These are structures where charge carriers are confined in all three spatial dimensions. The dot size, typically between $10 \mathrm{~nm}$ and $1 \mu \mathrm{m}$ [8], is on the order of the Fermi wavelength in the host material. The confinement of the quantum dots is usually achieved by electrical gating of a two-dimensional electron gas (2DEG), possibly combined with etching techniques, see Figs. 10 2(a), and 4(b). Small dots have charging energies in the meV range, resulting in quantization of charge on the dot (Coulomb blockade). This allows precise control of the number of electrons and of the spin ground state on the dot. Such a control of the number of electrons in the conduction band of a quantum dot (starting from zero) has been achieved with GaAs heterostructures, e.g., for vertical dots [11] and lateral dots [12, 13]. Quantum dots have various tunable parameters. These include geometry, energy spectrum, coupling between dots, etc., which open up many possibilities by providing a versatile system for manipulation of electronic states, in particular the spin state. Further, the electronic dot-orbitals are highly sensitive to external magnetic and electric fields [8, 11], since the magnetic length corresponding to fields of $B \approx 1 \mathrm{~T}$ is comparable to typical dot sizes. In coupled quantum dots, Coulomb blockade effects [14, tunneling between neighboring dots [8, 14, 15], and magnetization [16] have been observed as well as the formation of a delocalized single-particle state [17, 18, and coherent charge oscillations [19]. 


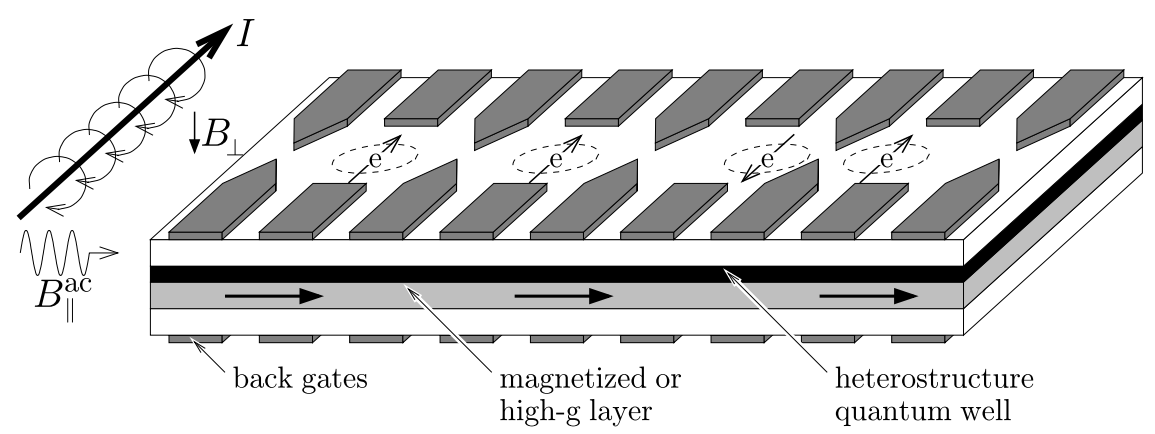

Figure 1. An array of quantum dots (circles) is defined by gate electrodes (dark gray) which confine the electrons. For spin manipulations, electrons can be moved by changing the gate voltage, pushing the electron wave function into the magnetized or high- $g$ layer, allowing for spatially varying Zeeman splittings. Alternatively, local magnetic fields can be achieved by a current-carrying wire (indicated on the left of the dot array). Then, the electron in each dot is subject to a distinct Zeeman splitting. This can be used for one-qubit gates, since only relative spin rotations are sufficient. Further, the spins can be addressed individually with ESR pulses of an oscillating in-plane magnetic field which is in resonance with a particular Zeeman splitting. These mechanisms allow single-spin rotations in different spatial directions. For gate operations on two qubit spins, their exchange coupling can be controlled by lowering the tunnel barrier between the dots, see Sec. 2] Here, the two rightmost dots are drawn schematically as tunnel-coupled. Note that only electrical switching is required to control spin dynamics and quantum computation with such a device.

\subsection{Quantum Computing with Spin Qubits}

The interest in quantum computing [20, 21] derives from the hope to outperform classical computers using new quantum algorithms. These algorithms make use of the quantum computer's abilities to exist in a quantum superpositions of its "binary" basis states $|0 \cdots 00\rangle,|0 \cdots 01\rangle$, $|0 \cdots 10\rangle, \ldots$, and to perform unitary time evolutions $U\left|\Psi_{\text {in }}\right\rangle=\left|\Psi_{\text {out }}\right\rangle$ for computation. The basis states can be realized by concatenating several quantum bits (qubits) which are states in the Hilbert space spanned by $|0\rangle$ and $|1\rangle$. A natural candidate for the qubit is the electron spin because every spin $\frac{1}{2}$ encodes exactly one qubit. Such spin qubits on quantum dots are good candidates for realizing a quantum computer [9]. We consider the five criteria of DiVincenzo's checklist 22] which must all be satisfied for any physical implementation of a quantum computer. We briefly discuss that these criteria are satisfied for spins qubits [9, 23. These criteria provides us with a good starting point for going into the details of concrete parts of the actual implementation of spin qubits. In the following sections we then show where specific theories and current experiments give new insight into the realization of spin qubits. 
i) A scalable system with well characterized qubits is required. To speed up calculations using a quantum computer, one needs a large number of qubits, i.e., on the order of $10^{5}$. This requirement is achievable for spin qubits, since producing arrays of quantum dots is feasible with state-of-the-art techniques for defining nanostructures in semiconductors. Further, the electron's spin $\frac{1}{2}$ provides a natural qubit, setting $|0\rangle \equiv|\uparrow\rangle$ and $|1\rangle \equiv|\downarrow\rangle$.

ii) The state of the qubits must be initialized to a known value at the beginning of a computation. To initialize spin qubits, one can apply a large magnetic field $g \mu_{B} B \gg k T$ that allows them to relax to the thermal ground state. Alternatively, one can inject polarized electrons into the dot by using spin-polarizing materials [4, 5] or by using a spin filter [24] which we describe in Sec. 4.

iii) Long decoherence times, much longer than the gate operation time, is the most difficult criterion to satisfy for many quantum computer proposals. Here, the current knowledge about the spin qubits is very promising. Gate operation times well below one ns are in principle feasible [23]. Using theoretical estimates and experimental data on spin flip times, the expected decoherence times can reach ms, see Sec. 3 . Thus, the decoherence times could be eight orders of magnitude larger than the gate operation times.

iv) With a universal set of quantum gates, any quantum algorithm can be implemented by controlling a particular unitary evolution of the qubits. It is sufficient to have single-qubit gates and a universal twoqubit gate (e.g., XOR or square root of SWAP). Single qubit gates can be produced by controlling the local magnetic field, the local $g$ factor (or $g$ tensor), or local Overhauser field, which, e.g., can be achieved with a semiconductor heterostructure and electrical gating [23, 25, 26], see Fig. 1. To build two-qubit gates, one can use the exchange interaction which arises when two neighboring dots are tunnel coupled, which can again be controlled via gate voltages [9, 27]. We describe the exchange interaction of coupled dots in Sec. 2.

v) Qubit read out determines the result at the end of the computation by measuring specific qubits. There are several proposals for measuring the spin in quantum dots, most of them rely on transferring the information from the spin to the charge state [9], e.g., by making use of the Pauli principle [24, 28, 29], via the spin-orbit interaction [30], or by making use of the Zeeman splitting [29]. We discuss concrete read-out schemes for spin qubits in Sec. [5 and address experiments [31 where single-shot read out has been achieved. 


\section{Two Coupled Quantum Dots as Quantum Gates}

We now consider a pair of spin qubits which are coupled by the exchange interaction, which results from the combination of the Coulomb interaction and the Pauli exclusion principle. Two electrons in coupled quantum dots and in the absence of a magnetic field have a spin-singlet ground state, while the first excited state in the presence of sufficiently strong Coulomb repulsion is a spin triplet. Higher excited states are separated from these two lowest states by an energy gap, given either by the Coulomb repulsion or the single-particle confinement. The low-energy dynamics of such a system is described by the effective Heisenberg spin Hamiltonian,

$$
H_{\mathrm{S}}(t)=J(t) \mathbf{S}_{1} \cdot \mathbf{S}_{2}
$$

where $J(t)$ describes the exchange coupling between the two spins $\mathbf{S}_{1}$ and $\mathbf{S}_{2}$ and is given by the energy difference between the triplet and the singlet, $J=E_{T_{0}}-E_{S}$. After a pulse of $J(t)$ with $\int_{0}^{\tau_{s}} d t J(t) / \hbar=\pi(\bmod$ $2 \pi)$, the time evolution $U(t)=T \exp \left(i \int_{0}^{t} H_{\mathrm{S}}(\tau) d \tau / \hbar\right)$ corresponds to the SWAP operator $U_{\mathrm{SW}}$, whose application leads to an interchange of the two spin states. While $U_{\mathrm{Sw}}$ is not sufficient for quantum computation, any of its square roots, say $U_{\mathrm{sw}}^{1 / 2}|\phi \chi\rangle=(|\phi \chi\rangle+i|\chi \phi\rangle) /(1+i)$, turns out to be a universal quantum gate. It can be used, together with single-qubit rotations, to assemble any quantum algorithm [9].

We consider a system of two coupled quantum dots in a 2DEG, containing one (excess) electron each, see Fig. 2(a). The dots are arranged in a plane such that the electrons can tunnel between the dots, leading to an exchange interaction $J$ between the two spins, which we now calculate. We model this system of coupled dots with the Hamiltonian $H=\sum_{i=1,2} h_{i}+C+H_{\mathrm{Z}}=H_{\text {orb }}+H_{\mathrm{Z}}$. The single-electron dynamics in the 2DEG ( $x y$-plane) is defined with the Hamiltonian $h_{i}$, containing the quartic confinement potential

$$
V(x, y)=\frac{m \omega_{0}^{2}}{2}\left[\frac{1}{4 a^{2}}\left(x^{2}-a^{2}\right)^{2}+y^{2}\right]
$$

with inter-dot distance $2 a$, effective Bohr radius $a_{\mathrm{B}}=\sqrt{\hbar / m \omega_{0}}$, and effective mass $m$. Separated dots $\left(a \gg a_{\mathrm{B}}\right)$ are thus modeled as two harmonic wells with frequency $\omega_{0}$, consistent with experiments where the low-energy spectrum of single dots indicates a parabolic confinement [11. A magnetic field $\mathbf{B}=(0,0, B)$ is applied along the $z$-axis, which couples to the electron spins through the Zeeman interaction $H_{Z}$ and to the charges through the vector potential $\mathbf{A}(\mathbf{r})=\frac{B}{2}(-y, x, 0)$. In almost depleted regions, like few-electron quantum dots, the screening length $\lambda$ can be expected to be much larger than the screening length 
(a)

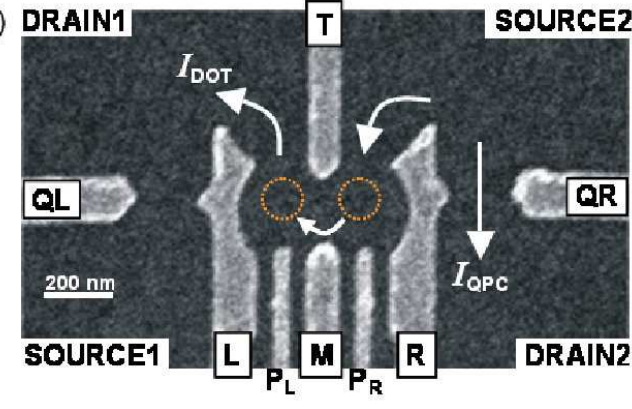

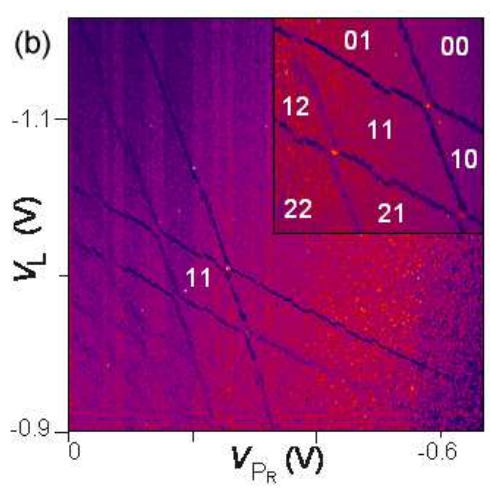

Figure 2. (a) Double dot structure with a single electron in each dot, shown as scanning electron micrograph of the metallic surface gates [12]. The circles indicate the two quantum dots and the arrows show the possible current paths. A bias voltage, $V_{\text {DOT }}$, can be applied between source 2 and drain 1, leading to current through the dots. A bias voltage, $V_{\mathrm{SD} i}$ between source and drain $i=1,2$ yields a current, $I_{\mathrm{QPC}}$, through the corresponding QPC. (b) Charge stability diagram (honeycomb) 15. of the double quantum dot, measured with QPC-R [12. A modulation (0.3 $\mathrm{mV}$ at $17.77 \mathrm{~Hz})$ is applied to gate $\mathrm{L}$, and $d I_{\mathrm{QPC}} / d V_{\mathrm{L}}$ is measured with a lock-in amplifier and plotted versus $V_{\mathrm{L}}$ and $V_{\mathrm{PR}}$. The bias voltages are $V_{\mathrm{SD} 2}=100 \mu \mathrm{V}$ and $V_{\mathrm{DOT}}=V_{\mathrm{SD} 1}=0$. The inset shows a magnification of the honeycomb pattern for the first few electrons in the double dot. The labels " $n_{\mathrm{L}} n_{\mathrm{R}}$ " indicate the number of electrons in the left and right dot, and the double dot is completely empty in the region " 00. ."

in bulk 2DEG regions (where it is $40 \mathrm{~nm}$ for GaAs). Thus, for small quantum dots, say $\lambda \gg 2 a \approx 40 \mathrm{~nm}$, we consider the bare Coulomb interaction $C=e^{2} / \kappa\left|\mathbf{r}_{1}-\mathbf{r}_{2}\right|$, where $\kappa$ is the static dielectric constant.

Now we consider only the two lowest orbital eigenstates of $H_{\text {orb }}$, leaving us with one symmetric (spin singlet) and one antisymmetric (spin triplet) orbital state. The spin state for the singlet is $|S\rangle=(|\uparrow \downarrow\rangle-$ $|\downarrow \uparrow\rangle) / \sqrt{2}$, while the triplet spin states are $\left|T_{0}\right\rangle=(|\uparrow \downarrow\rangle+|\downarrow \uparrow\rangle) / \sqrt{2}$, $\left|T_{+}\right\rangle=|\uparrow \uparrow\rangle$, and $\left|T_{-}\right\rangle=|\downarrow \downarrow\rangle$. For $k T \ll \hbar \omega_{0}$, higher-lying states are frozen out and $H_{\text {orb }}$ can be replaced by the effective Heisenberg spin Hamiltonian [Eq. (11)]. To calculate the triplet and singlet energies, we use the analogy between atoms and quantum dots and make use of variational methods similar to the ones in molecular physics. Using the Heitler-London ansatz with the ground-state single-dot orbitals, we find [27,

$$
\begin{aligned}
J= & \frac{\hbar \omega_{0}}{\sinh \left(2 d^{2} \frac{2 b-1}{b}\right)}\left\{\frac{3}{4 b}\left(1+b d^{2}\right)\right. \\
& \left.+c \sqrt{b}\left[e^{-b d^{2}} I_{0}\left(b d^{2}\right)-e^{d^{2}(b-1 / b)} I_{0}\left(d^{2}(b-1 / b)\right)\right]\right\},
\end{aligned}
$$



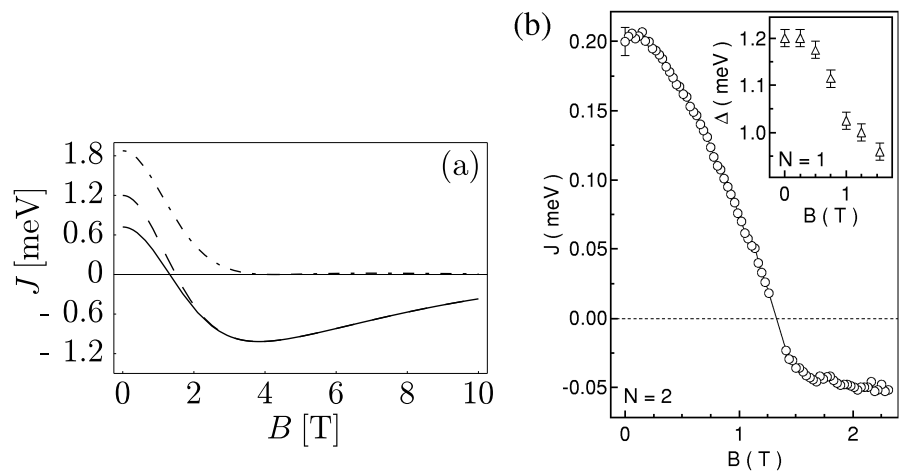

Figure 3. The exchange coupling $J$ (solid line) for GaAs quantum dots a function of the magnetic field $B$. (a) Theoretical prediction [Eq. (3)] for a double dot with confinement energy $\hbar \omega=3 \mathrm{meV}$, inter-dot distance $d=a / a_{\mathrm{B}}=0.7$, and $c=2.42$ [27]. For comparison, the short-range Hubbard result $J=4 t^{2} / U$ (dashed-dotted line) and the extended Hubbard result $J=4 t^{2} / U+V$ (dashed line) are plotted. (b) Experimentally observed exchange coupling $J$ via transport measurements 33 . Although a single dot structure was used, the measurements show double dot features, indicating that a double dot is formed within the structure. The dependence on magnetic field $B$ is in agreement with the theoretical predictions, in particular, $J$ can be tuned through zero near $B=1.3 \mathrm{~T}$.

with zeroth order Bessel function $I_{0}$, dimensionless distance $d=a / a_{\mathrm{B}}$ between the dots, magnetic compression factor $b=\sqrt{1+\omega_{L}^{2} / \omega_{0}^{2}}$, and Larmor frequency $\omega_{L}=e B / 2 m c$. In Eq. (3), the first term arises from the confinement potential, while the terms proportional to the parameter $c=\sqrt{\pi / 2}\left(e^{2} / \kappa a_{\mathrm{B}}\right) / \hbar \omega_{0}$ result from the Coulomb interaction $C$; the exchange term is recognized by its negative sign. We are mainly interested in the weak coupling limit $\left|J / \hbar \omega_{0}\right| \ll 1$, where the groundstate Heitler-London ansatz is self-consistent. We plot $J(B)$ [Eq. (3)] in Fig. 3(a) and observe the singlet-triplet crossing, where the sign of $J$ changes from positive to negative (for the parameters chosen in Fig. 3(a) at $B \approx 1.3 \mathrm{~T})$. Finally, $J$ is suppressed exponentially, $\propto \exp \left(-2 d^{2} b\right)$, either by compression of the electron orbitals through large magnetic fields $(b \gg 1)$, or by large distances between the dots $(d \gg 1)$, where in both cases the orbital overlap of the states in the two dots is reduced. The Heitler-London result [Eq. (3)] was refined by taking higher levels and double occupancy of the dots into account (implemented in a Hund-Mullikan approach), which leads to qualitatively similar results [27, in particular concerning the singlet-triplet crossing. These results have been confirmed by numerical calculations which take more single-particle levels into account 32 .

A characterization of a double dot can be performed with transport measurements. We describe transport through a double quantum dot, 
using a master equation approach [34]. We calculate differential conductance $G=d I / d V_{\mathrm{SD}}$ as a function of the bias voltage $V_{\mathrm{SD}}=\Delta \mu / e$ in the sequential tunneling and cotunneling regime. We obtain the main peak of the Coulomb blockade diamond and its satellite peaks. Since the positions of these peaks are related to the interdot tunnel splitting and to the singlet-triplet splitting $J$, one can determine these values in a standard transport experiment. Further, our model can be checked independently, since we also predict which satellite peaks have positive or negative values of $G$ and since we describe structures inside the Coulomb blocked diamonds which are due to a combined effect of cotunneling and sequential tunneling [34]. When we measure transport properties of a structure resembling a single dot, we observe features as would be expected for a double dot [33]. This indicates that a double dot in formed within our structure. We can then extract the $B$-dependent exchange coupling from our data which again is in agreement with theoretical predictions for double dots, see Fig. 3(b). That singlet-triplet crossings occur in single dots is established experimentally [35.

In further experiments, we measured a double quantum dot with tunable tunnel couplings. Spectroscopy of the double dot states was performed using a quantum point contact (QPC) as a local charge sensor. From the charge distribution on the double dot, we can deduce charge delocalization as a function of temperature and strength of tunnel coupling. Conversely, we can measure the tunnel coupling $t$ as function of the voltage applied on a gate in the coupling region. We find that the tunneling coupling is tunable from $t=0$ to $t=22 \mu \mathrm{eV}$ when the gate voltage is increased [18.

For few-electron quantum dots, the charging energies of a double quantum dot can be tuned such that there is only a single electron in each dot. The number of electrons on the dots can be controlled by simultaneously measuring the charge distribution with a QPC charge sensor [12, see Fig. 2] or by measuring transport through the double $\operatorname{dot}[36]$.

\section{Spin relaxation}

The lifetime of an electron spin is described by the following two time scales. The (longitudinal) spin relaxation time $T_{1}$ describes the time scale of a spin-flip process when the electron is aligned along the ex-

ternal magnetic field. The spin decoherence time $T_{2}$ is the lifetime of a coherent superposition $\alpha|\uparrow\rangle+\beta|\downarrow\rangle$. Since quantum gate operations require coherence of the underlying qubits, they must be carried out on times shorter than $T_{2}$. We note that $T_{2} \leq 2 T_{1}$ and typically even 
$T_{2} \ll T_{1}$ [37], thus from the sole knowledge of $T_{1}$, no lower bound for $T_{2}$ can be deduced. Therefore, it is of interest to investigate the interactions leading to decoherence (as we do now) and to find ways of measuring the decoherence time $T_{2}$ in an experiment (see Sec. 6).

For spins on quantum dots, one possible source of spin relaxation and decoherence is spin-orbit interaction. Calculations show that phononassisted spin-flip times [38, 39] in quantum dots are unusually long. This is so because the spin-orbit coupling in two-dimensions (2D) is linear in momentum, both for Dresselhaus and Rashba contributions. Due to this linearity, the effective magnetic field due to spin-orbit fluctuates transversely to the external magnetic field (in leading order). This implies that $T_{2}=2 T_{1}$ for spin-orbit interaction [40] and thus long decoherence times are expected. Another source of decoherence is the hyperfine coupling between electron spin and nuclear spins in a quantum dot [27, 41, 42], since all naturally occurring Ga and As isotopes have a nuclear spin $I=3 / 2$. It is known that such decoherence can be controlled by a large magnetic field or by polarizing the nuclear spins, i.e., by creating an Overhauser field [27.

The spin relaxation time $T_{1}$ of single electron spins on quantum dots was measured in recent experiments. One way to assess $T_{1}$ is to measure transport through the dot while applying double-step pulses to the gate voltage of the dot. First, the dot is emptied and filled again with one electron with a random spin. Then, the electron is held in the dot during a time $t_{h}$. Finally, the gate voltage is tuned such that the electron can tunnel out of the dot and contribute to a current, but only if it is in the excited spin state. Thus, the (time-averaged) current will proportional to the probability of having an excited spin on the dot after time $t_{h}$; this probability decays on the time scale of $T_{1}$. In these experiments, the limited current sensitivity puts an upper bound on $t_{h}$. Since $T_{1}$ turned out to be longer than this bound, one was not able to measure $T_{1}$. Still, it is possible to obtain a lower bound of for $T_{1}$ and $\approx 100 \mu$ s was obtained for triplet to singlet transitions [43] and for $N=1$ Zeeman levels [44]. Using a charge read-out device (see Sec. 5 ), single tunneling events can be observed. This allowed us to measure $T_{1}$ directly and $T_{1}^{\exp }=1 \mathrm{~ms}$ was obtained at $B=8 \mathrm{~T}$ [31. We now compare this value with theoretical predictions [40]. We assume a GaAs dot with Dresselhaus spin-orbit interaction $H_{\mathrm{SO}}=\beta\left(-p_{x} \sigma_{x}+p_{y} \sigma_{y}\right)$, with quantum well thickness $d=5 \mathrm{~nm}$, and with lateral size quantization energy $\hbar \omega_{0}=1.1 \mathrm{meV}$, corresponding to a Bohr radius $a_{\mathrm{B}}=32 \mathrm{~nm}$. The material parameters are the dielectric constant $\kappa=13.1$, coupling constant of deformation potential $\Xi_{0}=6.7 \mathrm{eV}$, piezoelectric constant $h_{14}=-0.16 \mathrm{C} / \mathrm{m}^{2}$, sound velocity $s_{j}$ for branch $j$, namely $s_{1}=4.73 \times 10^{5} \mathrm{~cm} / \mathrm{s}$ and $s_{2}=s_{3}=3.35 \times 10^{5} \mathrm{~cm} / \mathrm{s}$, sample density $\rho_{c}=5.3 \times 10^{3} \mathrm{~kg} / \mathrm{m}^{3}$, and 
effective mass $m^{*}=0.067 m_{e}$. The remaining unknown parameter is the spin-orbit length $\lambda_{\mathrm{SO}}=\hbar / m^{*} \beta$. It can be extracted from (independent) weak antilocalization measurements [45], where $\lambda_{\mathrm{SO}} \approx 9 \mu \mathrm{m}$ was found. Taking the Zeeman splitting used in the measurement of $T_{1}^{\exp }$, we obtain [40] $T_{1, \mathrm{SO}}^{\mathrm{th}} \approx 750 \mu \mathrm{s}$, with an error of $50 \%$ due to the uncertainty of the value of the Zeeman splitting. There is some additional uncertainty on the value of $\lambda_{\mathrm{SO}}$ which depends on electron density and growth of the sample. For example, we find $\lambda_{\mathrm{SO}} \approx 17 \mu \mathrm{m}$ in other samples [46], which would indicate a longer $T_{1}$ time since $T_{1} \propto \lambda_{\text {SO }}^{2}$ [40]. Within these uncertainties we find an agreement between experiments and theory, $T_{1}^{\mathrm{exp}} \approx T_{1, \mathrm{SO}}^{\mathrm{th}}$. Moreover, the predicted $B$-dependence [40] of $1 / T_{1}$ agrees well with the experiment [31], where a plateau is seen around $B \sim 10 \mathrm{~T}$. From this we can conclude that the spin-phonon mechanism is the dominant source for spin relaxation (and not hyperfine interaction). Since $T_{2}=2 T_{1}$ for spin-orbit interaction [40 and since there is no difference between decoherence and relaxation for hyperfine interaction [41, 42, we can expect spin decoherence times $T_{2}$ to be on the order of milliseconds.

\section{Spin Filter}

An important device for spintronics is a spin filter which selectively transmits electrons with respect to their spin orientation. For quantum computation with spin qubits, such a spin filter can be used for initialization and read out, see Sec. 1.2 and 5 . We proposed to use a quantum dot attached to in- and outgoing current leads as a spin filter [24. The direction of polarization of this spin filter can be tuned electrically by changing the gate voltage on the quantum dot. We now describe the operational principle of such a spin filter and present experimental implementations [47, 48, 49].

Our spin filter proposal [24] requires a lifted spin-degeneracy on the dot with a Zeeman splitting $\Delta_{z}=\left|\mu_{B} g B\right|$. For two electrons on the dot, we assume a singlet ground state with energy $E_{S}$, while the lowest-lying triplet state has a higher energy $E_{T_{+}}$. Let us consider the sequential tunneling transition where the number of electrons on the dots changes from 1 to 2 . The bias between the leads at chemical potentials $\mu_{1,2}$ is $\Delta \mu=\mu_{1}-\mu_{2}>0$. For small bias and low temperatures such that $\Delta \mu, k T<\min \left\{\Delta_{z}, E_{T_{+}}-E_{S}\right\}$, only ground state transitions are energetically allowed, i.e., $|\uparrow\rangle \leftrightarrow|S\rangle$. Thus, only spin down electrons can tunnel through the dot, see Fig. 5(a). We calculate the current through the dot using the standard tunneling Hamiltonian approach in the Coulomb blockade regime [8] and the master equation for the 

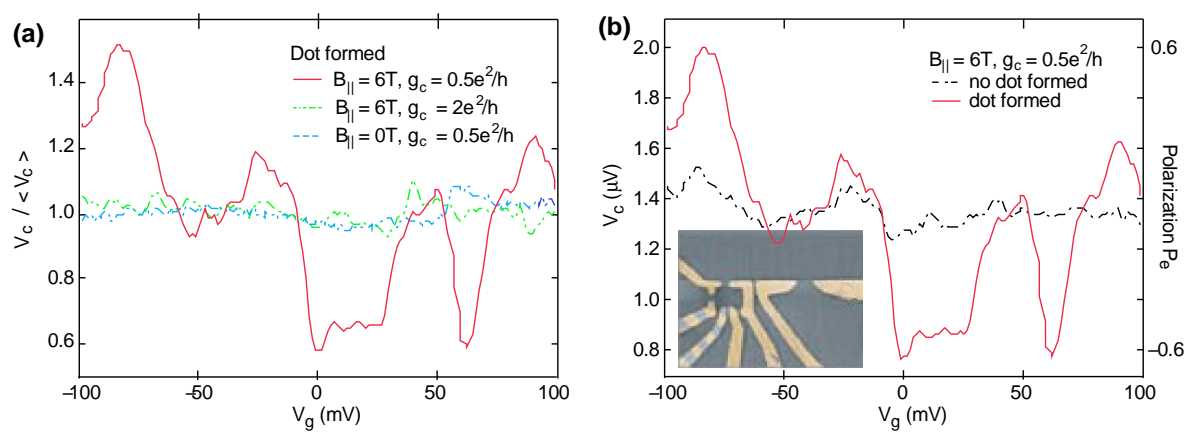

Figure 4. The spin polarization of current through a quantum dot is detected with an analyzer setup. The polarization is measured via the collector voltage (at the focusing peak, see text). The polarization of the current though the quantum dot in a magnetic field fluctuates as function of gate voltage. The fluctuations in the collector voltage only occur when the emitter forms a quantum dot, the collector is spin-sensitive, and an in-plane magnetic field is applied 48. (a) Comparison of normalized focusing peak height as a function of $V_{g}$ at $B_{\|}=6 \mathrm{~T}$ for a spin-selective collector, $g_{c}=0.5 e^{2} / h$ (red curve), at $B_{\|}=6 \mathrm{~T}$ for an unpolarized collector, $g_{c}=2 e^{2} / h$ (green curve), and at $B_{\|}=0$ with $g_{c}=0.5 e^{2} / h$ (blue curve). Dividing by average peak height, $\left\langle V_{c}\right\rangle$, normalizes for changes in focusing efficiency. (b) Focusing peak height at $B_{\|}=6 \mathrm{~T}$ with spin-selective collector, $g_{c}=0.5 e^{2} / h$, comparing an emitter which is a point contact at $2 e^{2} / h$ (black curve) and an emitter which is a quantum dot with both leads at $2 e^{2} / h$ (red curve). The inset shows a micrograph of the measured device, where the dot on the left and the QPC on the right side 48 .

reduced density matrix of the dot [24]. The current in first order in tunneling is the sequential tunneling current $I_{s}[8]$, which is spin- $\downarrow$ polarized. The second-order contribution is the cotunneling current $I_{c}$ [50] which involves a virtual intermediate state, where energy conservation can be violated for a short time. Thus, our energetic argument does not hold here and the cotunneling current $I_{c}$ contains a spin- $\uparrow$ component, reducing the efficiency of the spin-filtering effect. For $\Delta_{z}<E_{T_{+}}-E_{S}$, the ratio of spin-polarized to unpolarized current is [24]

$$
I_{s}(\downarrow) / I_{c}(\uparrow) \sim \frac{\Delta_{z}^{2}}{\left(\gamma_{1}+\gamma_{2}\right) \max \left\{k_{B} T, \Delta \mu\right\}},
$$

where $\gamma_{l}$ is the tunneling rate between lead $l$ and the dot. In the sequential tunneling regime we have $\gamma_{l}<k_{B} T, \Delta \mu$, thus, the ratio Eq. (4) is large and the spin-filter is efficient. We implemented this spin filter with a single quantum dot in the few electron regime [47]. The measured currents agree well with the theoretical predictions, see Fig. 15

Spin filtering properties of both open 48 and Coulomb blockaded [49] quantum dots were measured directly in a polarizer-analyzer geometry, where the spin polarization of current emitted from the dot (polarizer) was detected using a QPC at $g=0.5 e^{2} / h$ (analyzer) [51]. 


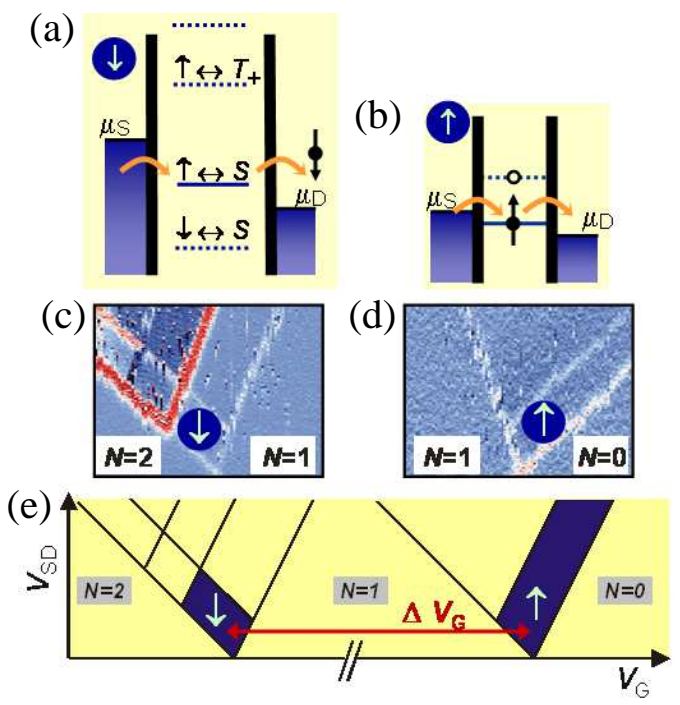

Figure 5. Spin filter in the sequential tunneling regime [24]. (a), (b) Operation principle of the spin filter. (a) Regime where the only allowed $1 \leftrightarrow 2$ electron transitions are $\uparrow \leftrightarrow S$ due to energy conservation, thus only spin- $\downarrow$ electron pass through the dot (see text). (b) The $0 \leftrightarrow 1$ electron transition provides a spin filter for spin- $\uparrow$ electrons. (c), (d) The experimentally measured $d I / d V_{\mathrm{SD}}$ is plotted as function of bias voltage $V_{\mathrm{SD}}$ and gate voltage $V_{\mathrm{G}}$ at $B_{\|}=12 \mathrm{~T}$ [47]. In the region labeled " $\downarrow$ " only spin-down electrons pass through the dot while in the region "个" only spin-up electrons. (e) Analyzing all transitions between the dot states $|0\rangle,|\uparrow\rangle$, $|\downarrow\rangle,|S\rangle,\left|T_{0}\right\rangle$, and $\left|T_{ \pm}\right\rangle$, the predicted $d I / d V_{\mathrm{SQ}}$ is shown schematically and agrees with the experimental data. This indicates that the current is spin polarized in the regimes labeled by arrows 47 .

These polarizer and analyzer elements were coupled by transverse focusing with the use of a small magnetic field applied perpendicular to the sample plane shown in the inset of Fig. 4(b). The collector voltage at the QPC shows a focusing peak when the distance between emitter and collector is an integer multiple of the cyclotron diameter. Measuring at the focusing peak, we find that in the presence of an in-plane field of a few Tesla or more, the current through the quantum dot (which is strongly coupled to leads) is indeed spin polarized. For the case of open dots [48], the direction of polarization can be readily tuned from along to against the applied in-plane field, see Fig. 4. However, for the closed dots, reversed spin filtering was not observed though ground-state peak motion was seen [49. More work is needed to clarify this departure from expectation. 


\section{Read-Out of a Single Spin}

At the end of every (quantum) computation, one reads out the result of the computation. For this it is sufficient to determine the state of some qubits which are either in state $|\uparrow\rangle$ or in state $|\downarrow\rangle$ (we do not need to measure a coherent superposition). However, it is very hard to detect an electron spin by directly coupling to its tiny magnetic moment (on the order of $\mu_{B}$ ). This difficulty is overcome by converting the spin information into charge information, which is then measured (we describe implementations below). Ideally, the qubit state can be determined in a single measurement, referred to as single shot read out. In general, however, there are some errors associated with the measurement, thus the preparation and measurement of the qubit need to be performed not only once but $n$ times. We now determine $n$ by assuming that the measurement has two possible outcomes, $A_{\uparrow}$ or $A_{\downarrow}$. Then, for an initial qubit state $|\uparrow\rangle$, with probability $p_{\uparrow}$ the outcome is $A_{\uparrow}$, which we would interpret as "qubits was in state $|\uparrow\rangle$." However, with probability $1-p_{\uparrow}$, the outcome is $A_{\downarrow}$ and one might incorrectly conclude that "qubit was in state $|\downarrow\rangle$ ". Conversely, the initial state $|\downarrow\rangle$ leads with probability $p_{\downarrow}$ to $A_{\downarrow}$ and with $1-p_{\downarrow}$ to $A_{\uparrow}$. How many times $n$ do the preparation of a qubit in the same initial state and subsequent measurement need to be performed until the state of the qubit is known with some given infidelity $\alpha$ ( $n$-shot read out)? We model the read out process with a positive operator valued measure (POVM) and find from a statistical analysis that we need [29]

$$
\begin{aligned}
& n \geq z_{1-\alpha}^{2}\left(\frac{1}{e}-1\right), \\
& e=\left(\sqrt{p_{\uparrow} p_{\downarrow}}-\sqrt{\left(1-p_{\uparrow}\right)\left(1-p_{\downarrow}\right)}\right)^{2},
\end{aligned}
$$

with the quantile (critical value) $z_{1-\alpha}$ of the standard normal distribution function, $\Phi\left(z_{1-\alpha}\right)=1-\alpha=\frac{1}{2}\left[1+\operatorname{erf}\left(z_{1-\alpha} / \sqrt{2}\right)\right]$. We interpret $e$ as measurement efficiency [29], since it is a single parameter $e \in[0,1]$ which tells us if $n$-shot read out is possible. For $p_{\uparrow}=p_{\downarrow}=1$, the efficiency is maximal, $e=100 \%$, and single-shot read out is possible $(n=1)$. When the measurement outcome is independent of the qubit state, i.e., $p_{\downarrow}=1-p_{\uparrow}$ (e.g., $p_{\uparrow}=p_{\downarrow}=\frac{1}{2}$ ), the state of the qubit cannot be determined and the efficiency is $e=0 \%$. For the intermediate regime, $0 \%<e<100 \%$, the state of the qubit is known after several measurements, with $n$ satisfying Eq. (5). In the more general case, the state of a register with $k$ different qubits should be determined with infidelity $\beta$. The probability that the state of all qubits is determined correctly is $1-\beta=(1-\alpha)^{k}$. One could expect that the required $n$ grows 
dramatically with $k$. Fortunately this is not the case, from Eq. (5) we find that $n \geq 2(1 / e-1) \log k / \beta$ is sufficient.

For the actual implementation of the spin qubit read out, the most prominent idea is to transfer the qubit information from spin to charge [9, 24, 28, 29, 31, 52, 53, which can then be accessed experimentally with sensitive voltage or current measurements. A straightforward concept yielding a potentially $100 \%$ reliable measurement requires a "spin-filter" 24 which allows only, say, spin-up but no spin-down electrons to pass through, as it is described in Sec. 4. For performing a measurement of a spin in a quantum dot, the spin filter is connected between this dot and a second ("reference") dot. The charge distribution on this system can be detected with sensitive electrometers [54 by coupling the dots to a quantum point contact [12, 55] or to a singleelectron transistor (SET) [56]. Then, if the spin had been up, it would pass through the spin filter into the second dot and a change in the charge distribution would be measured, while there is no change for spin down 9. Instead of a spin filter, one can use different Zeeman splittings on qubit and reference dot or make use the Pauli principle to read out the spin qubit via charge detection [29].

Finally, we consider the qubit dot coupled to a lead instead of a reference dot. For Zeeman splittings larger than temperature, one can tune the dot levels such that only the excited spin state, $|\downarrow\rangle$, can tunnel into the leads 28] with rate $\gamma_{\text {out }}$ (spin $\uparrow$ electrons can tunnel only onto the dot). Such a tunneling event changes the number of electrons on the dot and produces a pulse in the QPC current, whose duration must exceed $t_{\mathrm{m}}$ to be detected, until a spin $\uparrow$ electron tunnels onto the dot

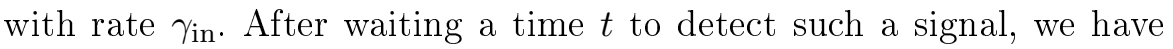
$p_{\uparrow}=1$ and $e=p_{\downarrow}=\left(1-e^{-t \gamma_{\text {out }}}\right) e^{-t_{\mathrm{m}} \gamma_{\text {in }}}$. We implemented this scheme experimentally [31]. Accounting also for finite $T_{1}$ and temperature, we obtain $p_{\uparrow}=92 \%$ and $p_{\downarrow}=70 \%$. This means that the measurement efficiency is $e=41 \%$, which is already very close to single-shot read out. For example, after 16 measurements, one knows the state of a 10 qubit register with an error smaller than $10^{-4}$. Further, this single spin detection scheme made it possible to determine the $T_{1}$ times of electron spins on quantum dots [31, see Sec. 3.

\section{Detection of Single-Spin Decoherence}

As it was seen in Sec. 3, it is an important research goal to measure the decoherence time $T_{2}$ of single spins on quantum dots. For this, we now describe how to extract the decoherence time $T_{2}$ from the sequential tunneling current through a quantum dot, in the presence of an applied 
electron spin resonance (ESR) field producing spin-flips on the dot 28]. We assume that the Zeeman splitting on the dot is $g \mu_{B} B>\Delta \mu, k_{B} T$, while the Zeeman splitting in the leads is different, such that the effect of the ESR field on the leads can be neglected. This can be achieved, e.g., by using materials with different $g$-factors for the dot and the leads. We derive the master equation and find the stationary reduced density matrix of the quantum dot in the basis $|\uparrow\rangle,|\downarrow\rangle,|S\rangle$ (with corresponding energies $0=E_{\uparrow}<E_{\downarrow}<E_{S}$ ). We can assume that the triplet is higher in energy and does not contribute to the sequential tunneling current. In the regime $E_{S}>\mu_{1}>E_{S}-g \mu_{B} B>\mu_{2}$, the current is blocked in the absence of the ESR field due to energy conservation. We calculate the the stationary current and find [28]

$$
I(\omega) \propto \frac{V_{\downarrow \uparrow}}{\left(\omega-g \mu_{B} B\right)^{2}+V_{\downarrow \uparrow}^{2}},
$$

where the width of the resonance at $\omega=g \mu_{B} B$ is given by the total spin decoherence rate $V_{\downarrow \uparrow}=\left(W_{S \uparrow}+W_{S \downarrow}\right) / 2+1 / T_{2}$. Here, $W_{S \sigma}$ denotes the rate for the transition from the state $|\sigma\rangle=|\uparrow\rangle,|\downarrow\rangle$ to the singlet $|S\rangle$ due to electrons tunneling from the leads onto the dot. Therefore, the inverse of the observed line width $1 / V_{\downarrow} \uparrow$ represents a lower bound for the intrinsic single-spin decoherence time $T_{2}$. For finite temperatures and in the linear response regime $\Delta \mu<k T$, the current has roughly the standard sequential tunneling peak shape $\cosh ^{-2}\left[\left(E_{S}-E_{\downarrow}-\mu\right) / 2 k_{B} T\right]$ as a function of the gate voltage $V_{\text {gate }} \propto \mu=\left(\mu_{1}+\mu_{2}\right) / 2$, while the width of the resonance in Eq. (7) as a function of $\omega$ remains unaffected.

The spin of a quantum dot in the presence of an ESR field shows coherent Rabi oscillations. It is possible to observe these Rabi oscillations of a single spin via time-averaged currents when ESR pulses are applied. Then, the time-averaged current $\bar{I}\left(t_{p}\right)$ as a function of the pulse length $t_{p}$ exhibits the Rabi oscillations of the spin-state of the dot [28], see Fig. [6] Observing such Rabi oscillations of a single spin would be a significant achievement, since this implied an working implementation of a one qubit gate.

\section{Conclusions}

We described the basic requirements for building a quantum computer with spin qubits. We addressed several concrete implementation issues for spin qubits, namely coupling between quantum dots, spin relaxation and decoherence measurements, spin filter devices, and single-spin read out setups. For all these issues, we reviewed theoretical and experimen- 

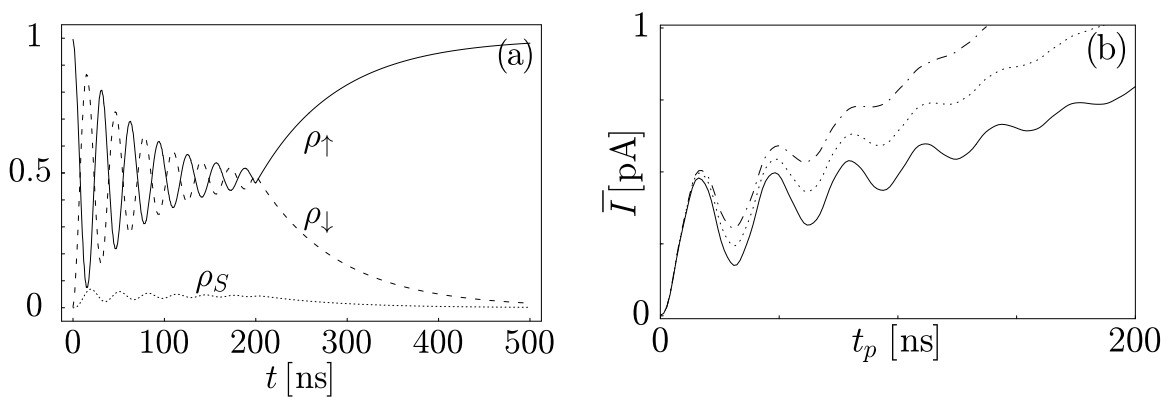

Figure 6. Single spin Rabi oscillations, generated by ESR pulses of length $t_{p}$, are observable in the time-averaged current $I\left(t_{p}\right)$ through a quantum dot 28. We take the amplitude of ESR field as $B_{x}^{0}=20 \mathrm{G}$ (and $g=2$ ), and $\Delta \mu>k T, \gamma_{1}=2 \times 10^{7} \mathrm{~s}^{-1}$, $\gamma_{2}=5 \gamma_{1}, T_{1}=1 \mu \mathrm{s}$, and $T_{2}=150 \mathrm{~ns}$. (a) Evolution of the density matrix $\rho$, where a pulse of length $t_{p}=200 \mathrm{~ns}$ is switched on at $t=0$, obtained via integration of master equation. (b) Time-averaged current $\bar{I}\left(t_{p}\right)$ (solid line) for a pulse repetition time $t_{r}=500 \mathrm{~ns}$. We also show the current where $\gamma_{1}$ and $\gamma_{2}$ are increased by a factor of 1.5 (dotted) and 2 (dash-dotted). Calculating the current contributions analytically, we obtain $\bar{I}\left(t_{p}\right) \propto 1-\rho_{\uparrow}\left(t_{p}\right)$, up to a background contribution $\bar{I}_{\mathrm{bg}}$ for times $t<t_{p}$, which is roughly linear in $t_{p}$. Thus, the current $\bar{I}$ probes the spin state of the dot at time $t_{p}$ and therefore allows one to measure the Rabi oscillations of a single spin [28].

tal results. These results give further insight in the details of quantum computing with spin qubits.

\section{Acknowledgments}

We acknowledge G. Burkard, L. DiCarlo, J.M. Elzerman, J.A. Folk, V. Golovach, R. Hanson, A. Khaetskii, R. Potok, P. Recher, L.M.K. Vandersypen, L.H. Willems van Beveren, and D. Zumbühl. This work was supported by ARO, DARPA, FOM, NCCR Nanoscience, and Swiss NSF.

\section{References}

1. G.A. Prinz, Phys. Today 45(4), 58 (1995); Science 282, 1660 (1998).

2. S.A. Wolf, D.D. Awschalom, R.A. Buhrman, J.M. Daughton, S. von Molnár, M.L. Roukes, A.Y. Chtchelkanova, and D.M. Treger, Science 294, 1488 (2001).

3. Semiconductor Spintronics and Quantum Computation, eds. D.D. Awschalom, D. Loss, and N. Samarth (Springer, Berlin, 2002).

4. R. Fiederling, M. Keim, G. Reuscher, W. Ossau, G. Schmidt, A. Waag, L.W. Molenkamp, Nature 402, 787 (1999).

5. Y. Ohno, D.K. Young, B. Beschoten, F. Matsukura, H. Ohno, D.D. Awschalom, Nature 402, 790 (1999). 
6. J.M. Kikkawa, I.P. Smorchkova, N. Samarth, and D.D. Awschalom, Science 277, 1284 (1997); J.M. Kikkawa and D.D. Awschalom, Phys. Rev. Lett. 80, 4313 (1998); D.D. Awschalom and J.M. Kikkawa, Phys. Today 52(6), 33 (1999).

7. J.A. Gupta, R. Knobel, N. Samarth, and D. D. Awschalom, Science 292, 2458 (2001).

8. L.P. Kouwenhoven, G. Schön, and L.L. Sohn, in Mesoscopic Electron Transport, Vol. 345 of NATO Advanced Study Institute, Series E, edited by L.L. Sohn, L.P. Kouwenhoven, and G. Schön (Kluwer Academic Publishers, Dordrecht, 1997).

9. D. Loss and D.P. DiVincenzo, Phys. Rev. A 57, 120 (1998); cond-mat/9701055

10. B.E. Kane, Nature 393, 133 (1998).

11. S. Tarucha, D. G. Austing, T. Honda, R. J. van der Hage, and L.P. Kouwenhoven, Phys. Rev. Lett. 77, 3613 (1996).

12. J.M. Elzerman, R. Hanson, J.S. Greidanus, L.H. Willems van Beveren, S. De Franceschi, L.M.K. Vandersypen, S. Tarucha, and L.P. Kouwenhoven, Phys. Rev. B 67, 161308R (2003).

13. M. Ciorga, A.S. Sachrajda, P. Hawrylak, C. Gould, P. Zawadzki, Y. Feng, and Z. Wasilewski, Physica E 11, 35 (2001).

14. F.R. Waugh, M.J. Berry, D.J. Mar, R.M. Westervelt, K.L. Campman, and A.C. Gossard, Phys. Rev. Lett. 75, 705 (1995); C. Livermore, C.H. Crouch, R.M. Westervelt, K.L. Campman, and A.C. Gossard, Science 274, 1332 (1996).

15. W.G. van der Wiel, S. De Franceschi, J.M. Elzerman, T. Fujisawa, S. Tarucha, and L.P. Kouwenhoven, Rev. Mod. Phys. 75, 1 (2003).

16. T.H. Oosterkamp, S.F. Godijn, M.J. Uilenreef, Y.V. Nazarov, N.C. van der Vaart, and L.P. Kouwenhoven, Phys. Rev. Lett. 80, 4951 (1998).

17. R.H. Blick, D. Pfannkuche, R.J. Haug, K. v. Klitzing, and K. Eberl, Phys. Rev. Lett. 80, 4032 (1998); ibid. 81, 689 (1998); T.H. Oosterkamp, T. Fujisawa, W.G. van der Wiel, K. Ishibashi, R.V. Hijman, S. Tarucha, L.P. Kouwenhoven, Nature 395, 873 (1998); I.J. Maasilta and V.J. Goldman, Phys. Rev. Lett. 84, 1776 (2000).

18. L. DiCarlo, H.J Lynch, A.C. Johnson, C.M. Marcus, M.P. Hanson, A.C. Gossard, Phys. Rev. Lett. 92, 226801 (2004).

19. T. Hayashi, T. Fujisawa, H.-D. Cheong, Y.-H. Jeong, Y. Hirayama, Phys. Rev. Lett. 91, 226804 (2003).

20. A. Steane, Rep. Prog. Phys. 61, 117 (1998).

21. M.A. Nielsen, I.L. Chuang, Quantum Computation and Quantum Information (Cambridge U. Press, New York, 2000).

22. D.P. DiVincenzo, Fortschr. Phys. 48, 771 (2000).

23. G. Burkard, H.-A. Engel, and D. Loss, Fortschr. Phys. 48, 965 (2000); G. Burkard and D. Loss, Ch. 8 in Ref. 3.

24. P. Recher, E.V. Sukhorukov, and D. Loss, Phys. Rev. Lett. 85, 1962 (2000).

25. G. Salis, Y. Kato, K. Ensslin, D.C. Driscoll, A.C. Gossard, and D.D. Awschalom, Physica E 16, 99 (2003).

26. Y. Kato, R.C. Myers, D.C. Driscoll, A.C. Gossard, J. Levy, and D.D. Awschalom, Science 299, 1201 (2003).

27. G. Burkard, D. Loss, and D. P. DiVincenzo, Phys. Rev. B 59, 2070 (1999).

28. H.-A. Engel, D. Loss, Phys. Rev. Lett. 86, 4648 (2001); Phys. Rev. B 65, 195321 (2002).

29. H.-A. Engel, V. Golovach, D. Loss, L.M.K. Vandersypen, J.M. Elzerman, R. Hanson, and L.P. Kouwenhoven, Phys. Rev. Lett. 93, 106804 (2004);

30. L.S. Levitov and E.I. Rashba, Phys. Rev. B 67, 115324 (2003). 
31. J.M. Elzerman, R. Hanson, L.H. Willems van Beveren, B. Witkamp, L.M.K. Vandersypen, and L.P. Kouwenhoven, Nature 430, 431 (2004).

32. X. Hu and S. Das Sarma, Phys. Rev. A 61, 062301 (2000).

33. D.M. Zumbühl, C.M. Marcus, M.P. Hanson, and A.C. Gossard, cond-mat/0408276

34. V.N. Golovach and D. Loss, Phys. Rev. B 69, 245327 (2004).

35. B. Su, V.J. Goldman, J.E. Cunningham, Phys. Rev. B 46, 7644 (1992); R.C. Ashoori, H.L. Stormer, J.S. Weiner, L.N. Pfeiffer, K.W. Baldwin, and K.W. West, Phys. Rev. Lett. 71, 613 (1993); T. Schmidt, M. Tewordt, R.H. Blick, R.J. Haug, D. Pfannkuche, and K. v. Klitzing, Phys. Rev. B 51, 5570 (1995).

36. I.H. Chan, P. Fallahi, A. Vidan, R.M. Westervelt, M. Hanson, A.C. Gossard, cond-mat/0309205

37. A. Abragam and B. Bleaney, Electron Paramagnetic Resonance of Transition Ions (Clarendon Press, Oxford, 1970).

38. A.V. Khaetskii and Y.V. Nazarov, Phys. Rev. B 64, 125316 (2001).

39. S.I. Erlingsson and Y.V. Nazarov, Phys. Rev. B 66, 155327 (2002).

40. V. Golovach, A. Khaetskii, and D. Loss, Phys. Rev. Lett. 93, 016601 (2004).

41. A.Khaetskii, D. Loss, and L. Glazman, Phys. Rev. Lett. 88, 186802 (2002).

42. A.Khaetskii, D. Loss, and L. Glazman, Phys. Rev. B 67, 195329 (2003).

43. T. Fujisawa, D.G. Austing, Y. Tokura, Y. Hirayama, S. Tarucha, Nature 419, 278 (2002).

44. R. Hanson, B. Witkamp, L.M.K. Vandersypen, L.H. Willems van Beveren, J.M. Elzerman, L.P. Kouwenhoven, Phys. Rev. Lett. 91, 196802 (2003).

45. D.M. Zumbühl, J.B. Miller, C.M. Marcus, K. Campman, and A.C. Gossard, Phys. Rev. Lett. 89, 276803 (2002). Note that their $\lambda_{\text {SO }}^{\prime}$ is related to ours by $\lambda_{\mathrm{SO}}=2 \lambda_{\mathrm{SO}}^{\prime}$.

46. A.G. Huibers, J.A. Folk, S.R. Patel, C.M. Marcus, C.I. Duruöz, and J.S. Harris, Jr., Phys. Rev. Lett. 83, 5090 (1999).

47. R. Hanson, L.M.K. Vandersypen, L.H. Willems van Beveren, J.M. Elzerman, I.T. Vink, and L.P. Kouwenhoven, cond-mat/0311414

48. J.A. Folk, R.M. Potok, C.M. Marcus, and V. Umansky, Science 299, 679 (2003).

49. R.M. Potok, J.A. Folk, C.M. Marcus, V. Umansky, M. Hanson, and A.C. Gossard, Phys. Rev. Lett. 91, 016802 (2003).

50. D.V. Averin and Yu. V. Nazarov, in Single Charge Tunneling, eds. H. Grabert and M.H. Devoret, NATO ASI Series B, Vol. 294, Plenum Press, New York, 1992.

51. R.M. Potok, J.A. Folk, C.M. Marcus, and V. Umansky, Phys. Rev. Lett. 89, 266602 (2002).

52. B.E. Kane, N.S. McAlpine, A.S. Dzurak, R.G. Clark, G.J. Milburn, H.B. Sun, and H. Wiseman, Phys. Rev. B 61, 2961 (2000).

53. M. Friesen, C. Tahan, R. Joynt, and M.A. Eriksson, Phys. Rev. Lett. 92, 037901 (2004).

54. M. Devoret, D. Estève, and Ch. Urbina, Nature (London) 360, 547 (1992).

55. M. Field, C. G. Smith, M. Pepper, D. A. Ritchie, J. E. F. Frost, G. A. C. Jones, and D. G. Hasko, Phys. Rev. Lett. 70, 1311 (1993).

56. W. Lu, Z.Q. Ji, L. Pfeiffer, K.W. West, and A.J. Rimberg, Nature 423 (6938), $422(2003)$. 